\title{
Research progress on KRAS mutations in colorectal cancer
}

\author{
Marco Cefali ${ }^{1, \#, ~ S a m a n t h a ~ E p i s t o l i o ~}{ }^{2, \#}$, Maria Celeste Palmarocchi ${ }^{1}$, Milo Frattini $i^{2, *}$, Sara De Dosso ${ }^{1,3,{ }^{*}}$ \\ 'Department of Medical Oncology, Oncology Institute of Southern Switzerland, Bellinzona 6500, Switzerland. \\ ${ }^{2}$ Institute of Pathology, Laboratory of Molecular Pathology, Locarno 6600, Switzerland. \\ ${ }^{3}$ Università della Svizzera Italiana, Lugano 6900, Switzerland. \\ \#Authors contributed equally. \\ "These authors are Joint Senior Authors.
}

Correspondence to: Dr. Sara De Dosso, Department of Medical Oncology, Oncology Institute of Southern Switzerland, Via A. Gallino 12, Bellinzona 6500, Switzerland. E-mail: sara.dedosso@eoc.ch

How to cite this article: Cefalì M, Epistolio S, Palmarocchi MC, Frattini M, De Dosso S. Research progress on KRAS mutations in colorectal cancer. J Cancer Metastasis Treat 2021;7:26. https://dx.doi.org/10.20517/2394-4722.2021.61

Received: 12 Mar 2021 First Decision: 19 Apr 2021 Revised: 20 Apr 2021 Accepted: 6 May 2021 Published: 11 May 2021

Academic Editor: Lucio Miele Copy Editor: Yue-Yue Zhang Production Editor: Yue-Yue Zhang

\begin{abstract}
The RAS gene family, responsible for signal transduction within the mitogen activated protein kinase (MAPK) and phosphatidylinositol 3 kinase (PI3K) pathways, is frequently involved in carcinogenesis, and alterations in its member genes can be detected, with variable frequency, in a wide variety of solid and hematological cancers. These alterations may behave as prognostic-predictive biomarkers and driver mutations, making them an interesting therapeutic target. Since their discovery, many strategies have been pursued to act on their signaling pathways. Indeed, in clinical practice, KRAS, the most prominent member of the RAS gene family, represents an especially elusive target in most malignancies; pathway inhibition is carried out upstream, on the EGFR receptor, or downstream, most frequently on the BRAF/MEK/ERK cascade. Recently, clinically relevant direct RAS inhibition has been successfully achieved with the development of potent and selective covalent inhibitors of KRAS c.34G>T (p.G12C). These latest-generation drugs represent both a new and interesting tool in the therapeutic armamentarium and a symbolic end to the myth of KRAS undruggability. However, their clinical relevance and appropriate place in treatment strategies remain to be determined.
\end{abstract}

Keywords: KRAS, targeted therapies, EGFR pathway, MAPK, colorectal cancer 


\section{INTRODUCTION}

Identification of the RAS gene family can be traced back to the 1970s, with the definition of the oncogenic sequences of the Kirsten and Harvey strains of murine sarcoma virus ${ }^{[1]}$. The human homologs of these genes, however, were not identified until the 1980s; HRAS and KRAS are homologs to the Harvey and Kirsten murine oncogenes, respectively, while NRAS was identified in a human neuroblastoma cell line $\mathrm{e}^{[2,3]}$. These genes encode for closely related GTPase proteins responsible for signal transduction within two main pathways, the mitogen-activated protein kinase (MAPK) and phosphoinositide-3 kinase pathways, involved in the regulation of cell growth, survival, and differentiation. In the scope of this review, we mainly focus on KRAS.

\section{ROLE OF KRAS IN COLORECTAL CANCER CARCINOGENESIS}

Cancer is the result of the gradual accumulation of gene mutations that lead to an increase of cell proliferation ${ }^{[4-6]}$. The evolution from benign to malignant lesions and their acquisition over time has been extensively studied, especially in colorectal cancer (CRC) ${ }^{[7]}$. Indeed, the so-called "Vogelgram", the most important model of cancer development, has been proposed in this tumor type. This model associates specific gene alterations to different stages of CRC development and identifies the occurrence of multiple, sequential, functional, and structural genetic alterations that, in the aggregate, cause a temporal progression from early lesions to late CRC formation ${ }^{[7-9]}$. The gatekeeper event is represented by point mutations that inactivate the APC gene in early phases (at the stage of adenoma), while those occurring in the TP53 gene are typically associated with late stages (carcinoma) or metastatic spreading ${ }^{[7,10]}$.

The core of the adenoma-carcinoma sequence is represented by point mutations in the KRAS gene that unleash a second round of clonal growth, which allows an expansion of cell numbers and, as a consequence, the evolution from a small to a large adenoma ${ }^{[7]}$. Overall, KRAS mutations provide only a small selective growth advantage to the cell, equal to a $0.4 \%$ increase in the difference between cell birth and cell death. Over many years, however, this slight increase can result in the development of a large KRAS mutant $\operatorname{mass}^{[6,7,9]}$.

KRAS is regulated by tyrosine kinase receptors and, in turn, activates the MAPK signaling pathway that is mainly involved in determining cell survival, leading to a strong promotion of cell growth and replication. In particular, mutations in KRAS gene confer to cancer cells the ability to grow in lower glucose concentrations than those required for the growth of normal cells or cancer cells that do not have mutations in these genes ${ }^{[1,12]}$.

A derivative of the "Vogelgram" is the involvement of KRAS in the phenomenon known as "chromosomal instability" (CIN), which is also considered to be the classical mechanism of CRC development since it represents the cause of up to $80 \%-85 \%$ of all CRC cases. CIN is characterized by imbalances in the number of chromosomes, thus leading to aneuploydic tumors and loss of heterozygosity ${ }^{[13]}$.

\section{MUTATION RATES IN A POPULATION-BASED COHORT OF COLORECTAL CANCER}

KRAS mutation rates are different among CRC patients belonging to different ethnicities. In Caucasians, the frequency of CRC with KRAS mutations is equal to $38 \%$; in Asians, it is close to $40 \%$; and, in Africans, it is only $21 \%^{[14,15]}$. Most KRAS mutations in CRC occur within KRAS exon 2, with prevalence ranging from $10 \%$ to $47 \%$, typically in codons 12 and 13 . In addition, approximately $10 \%$ of patients with CRC are characterized by other types of KRAS mutations, in exon 3 (in codons 59 and 61, accounting for about 2\%$3 \%$ of CRC) or exon 4 (in codons 117 and 146, with a mutation prevalence equal to $3 \%-4 \%$ ). In addition, the NRAS brother gene is also frequently altered, in a mutually exclusive manner with respect to KRAS 
mutations. NRAS is mutated in the same codons of KRAS (therefore, these positions are true hot-spot mutations), especially in exon 2 (observed in approximately $3 \%-5 \%$ of CRC) and exon 3 (2\%-6\% of CRC), while mutations in exon 4 are substantially absent ${ }^{[16,17]}$.

\section{KRAS in Caucasians}

In Europe, the most relevant KRAS exon 2 mutations in CRC are c.35G>A (p.G12D) (37\%), c.35G>T (p.G12V) (28\%), and c.38G>A (p.G13D) (15\%) changes ${ }^{[18]}$. However, there are important differences among European countries. For example, in Russia, there is a higher prevalence of c.35G>T (p.G12V) mutations (32.7\%) and a lower one of c.38G>A (p.G13D) mutations (12.5\%) in comparison with patients from other parts of Europe. Furthermore, in a geographically distinct region of Italy, Sardinia, mutation frequencies indicate a lower occurrence of the c.35G>A (p.G12D) change and a more frequent detection of other mutations in codon 12 [c.35G>A (p.G12D), 26\%; c.35G>T (p.G12V), 23\%; c.38G>A (p.G13D), 16\%; c.35G>C (p.G12A), 7\%; c.34G>T (p.G12C), 6\%; c.34G>A (p.G12S), 3\%; c.37G>T (p.G13C), 2\%; c.34G>C (p.G12R), 2\%; and c.38G>C/c.37G>A/c.38G>T (p.G13A/p.G13S/p.G13V), 2\%] ${ }^{[19]}$. These differences should be taken into account when assessing the response of CRC patients on chemotherapy and targeted therapy ${ }^{[20]}$. Concerning exons 3 and 4, the situation seems to be uniform across European countries, with the most common alterations [c.183A>C (p.Q61H), c.182A>T (p.Q61L), and c.182A $>$ G (p.Q61R)] in exon 3 accounting for $3 \%$ of the totality of KRAS mutant cases and the alterations in exon 4 [mainly c.436G>C (p.A146P), c.436G>A (p.A146T), and c.437C >T (p.A146V)] for $4 \%$ of KRAS mutations ${ }^{[19]}$.

\section{KRAS in Asians}

Concerning Asian populations, KRAS mutations are detected in 35\%-40\% of CRC cases in Chinese patients. In China, the mutations occur more commonly in codon 12 (79.1\%) and codon 13 (20.4\%) in exon 2, with c.35G >A (p.G12D) (37.8\%), c.35G>T (p.G12V) (20.0\%), and c.38G>A (p.G13D) (19.6\%) changes as the most frequent mutation types. These three point mutations account for 77.3\% (174/225) of KRAS gene mutations ${ }^{[2,22]}$. As a consequence, exons 3 and 4 mutations are less frequently detected, representing, $1 \%-2 \%$ and $4 \%-7 \%$ of the CRC population, respectively ${ }^{[23,24]}$. In particular, the most common mutations in exons 3 are c.183A>C (p.Q61H), c.182A>T (p.Q61L), c.182A>G (p.Q61R), characterizing 1.19\%, 0.74\%, and 0.15\% of the cases, respectively, while the most relevant mutations [c.436G>C (p.A146P), c.436G>A (p.A146T), c. $437 \mathrm{C}>\mathrm{T}$ (p.A146V), and c.351A $>\mathrm{C}(\mathrm{p} . \mathrm{K} 117 \mathrm{~N})]$ represent less than $4 \%$ of patients ${ }^{[23,25]}$.

In South Korea, the frequency of KRAS mutations is superimposable with that observed in China (37\%), mainly affecting codon $12(73.8 \%)$ and codon $13(24 \%)$. More in detail, the most frequent amino acid change is c.35G>A (p.G12D), which accounts for $36.9 \%$ of KRAS mutations, followed by c.38G $>A$ (p.G13D) $(24.2 \%)$ and c.35G>T (p.G12V) (21.9\%) ${ }^{[26]}$. Similarities between South Korea and China are also observed in mutation frequencies and type of mutations in KRAS exons 3 and $4^{[26]}$.

\section{KRAS in Africans}

Among continents, the most relevant difference of KRAS mutation rate in CRC has been observed in Africa. In Nigeria, the occurrence of KRAS exon 2 mutations (codons 12 and 13) has been described in only $21 \%$ of patients, meaning roughly half the rate of Caucasians and Asians ${ }^{[27]}$. Specific data concerning KRAS exon 3 and 4 mutations in the African population are still lacking.

\section{KRAS differences in the heterogeneous American population}

Another relevant difference in KRAS mutation rates has been observed in the comparison between African Americans and Caucasian Americans. KRAS mutations occurred significantly more frequently in African Americans than Caucasians ( $37 \%$ vs. $21 \%, P=0.01)$ and were associated with worse stage or grade of $\mathrm{CRC}^{[28]}$. It is noteworthy that such a difference is mainly caused by codon 12 mutations: indeed, many 
studies have reported that codon 12 mutations are significantly more frequent in African Americans than Caucasians $(P=0.04)$, whereas the frequencies of mutations in codon 13 , exon 3 , and exon 4 did not differ significantly according to ethnicity ${ }^{[27-29]}$. Interestingly, even within the same ethnic group, there can be differences. Namely, a higher rate of KRAS mutations has been reported in the Hispanic group (59\%) compared to the general Caucasian population $(38 \%)^{[15]}$. In addition, another relevant variability has been observed in Mexico: the KRAS mutation rate varies from $40 \%$ in the North Pacific cities to 59\% in the central Mexican region ${ }^{[30]}$. By comparing mutation rates between Africa and African Americans and among the different ethnicities in the United States, it is clear that, more than race (which is, for instance, important for the occurrence of EGFR mutations in lung adenocarcinomas), the aspects that seem to play a prominent role in the occurrence of KRAS mutations in CRC are environment and diet. In the United States, the dietary lifestyle of the less wealthy populations is often characterized by junk food meals and lower fiber and fruit intake. Compared to African populations, African Americans definitely have an excessive intake of calories and a higher consumption of red and processed meat.

\section{FREQUENCIES OF KRAS IN CRC AND OTHER CANCERS}

Mutations involving RAS genes are the most frequent oncogenic alterations in human cancers [Table 1] $]^{[31]}$. In particular, in the RAS family, KRAS is the most frequently mutated gene, followed by NRAS [Table 2] ${ }^{[31]}$. Overall, KRAS is altered in $15.95 \%$ of cancers, with pancreatic, lung, colon adenocarcinoma, colorectal, and rectal adenocarcinomas having the greatest prevalence of alterations ${ }^{[17]}$. In addition to CRC, KRAS oncogene activation through point mutations represents the main oncogene alteration in pancreatic cancer, where such an alteration is observed in more than $80 \%$ of cases. A percentage of alteration close to the one observed in CRC is found in lung adenocarcinomas and cholangiocarcinoma ${ }^{[32,33]}$ [Table 3].

The KRAS mutation pattern is tumor specific [Table 3] ${ }^{[17]}$. As mentioned above, in CRC, KRAS exon 2 mutations occur at an average rate of nearly $40 \%$; in particular, c.35G>A (p.G12D) is detected in $37 \%$ of cases; c.35G $>\mathrm{T}$ (p.G12V) in 29\%; c.34G>T (p.G12C) in 7\%; c.35G>C (p.G12A) in 9\%; c.34G>A (p.G12S) in $7 \%$; c.38G >A (p.G13D) in 14\%; and c.34G>C (p.G12R) in less than $1 \%$. Interestingly, in CRC, the most prevalent KRAS exon 2 mutations, c.35G>A (p.G12D) and c.35G>T (p.G12V), are weak drivers, whereas BRAF c.1799T>A (p.V600E) is a pivotal driver ${ }^{[18,34]}$. Compared to CRC, recent data define higher rates of KRAS exon 2 mutations in pancreatic tumors (79.7\%) and lower frequencies in non-small cell lung cancer (NSCLC) $(27.1 \%)^{[17]}$ [Table 3]. In particular, c.35G>A (p.G12D) mutation incidence is the highest in pancreatic cancer and the lowest in lung adenocarcinoma and c.35G>T (p.G12V) mutation is the most frequent in ovarian cancer and the least in cholangiocarcinoma. The most peculiar KRAS mutation is c. $34 \mathrm{G}>\mathrm{T}$ (p.G12C), which is by far the most frequent in lung adenocarcinoma (approximately $40 \%$ ) and significantly less frequent in other cancers (no more than 10\%). The other forms of mutation in exon 2 are much less prevalent but again related to specific cancer types: the c.35G>C (p.G12A) mutation characterizes endometrial cancer, the c.34G>A (p.G12S) mutation is cholangiocarcinoma specific, and the c.34G>C (p.G12R) mutation is typical of pancreatic cancer, while the c.38G>A (p.G13D) mutation is strictly associated with CRC, suggesting unique carcinogenic effects ${ }^{[18]}$. These mutational patterns are caused by various carcinogens and/or mutagenic processes, such as DNA repair deficiencies, and therefore are unique signatures of various cancer types ${ }^{[35]}$. For this reason, the result of the processing of red meat, producing O6methylguanine and heterocyclic aromatic amines compounds that produce bulky DNA adducts, which oftentimes cannot be removed from DNA due to nucleotide excision repair system errors, can be a critical issue. This situation can bring to the occurrence of KRAS (and APC) mutations in $\mathrm{CRC}^{\left[{ }^{[36}\right]}$. Furthermore, it has been demonstrated that the c.34G>T (p.G12C) change occurs predominantly in women affected by CRC upon exposure to acrylamide metabolites present in heat treated carbohydrate-rich foods ${ }^{[37]}$. 
Table 1. RAS mutations percentage detected in different types of cancers

\begin{tabular}{ll}
\hline Cancer & RAS mutations percentage \\
\hline Pancreatic Ductal Adenocarcinoma & $96 \%$ \\
CRC & $52 \%$ \\
Multiple myeloma & $43 \%$ \\
Lung adenocarcinoma & $32 \%$ \\
Skin cutaneous melanoma & $29 \%$ \\
\hline
\end{tabular}

CRC: Colorectal cancer (Hobbs et al. $\left.{ }^{[31]}, 2016\right)$.

Table 2. Specific KRAS, NRAS, and HRAS mutation percentages detected in different types of cancer

\begin{tabular}{llll}
\hline Cancer & $\begin{array}{l}\text { Rate of } \boldsymbol{K} \text { RAS in the total of } \\
\text { RAS mutations }\end{array}$ & $\begin{array}{l}\text { Rate of NRAS in the total of } \\
\text { RAS mutations }\end{array}$ & $\begin{array}{l}\text { Rate of HRAS in the total of } \\
\text { RAS mutations }\end{array}$ \\
\hline $\begin{array}{l}\text { Pancreatic ductal } \\
\text { adenocarcinoma }\end{array}$ & $100 \%$ & $0 \%$ & $0 \%$ \\
CRC & $86 \%$ & $14 \%$ & $0 \%$ \\
Multiple myeloma & $55 \%$ & $45 \%$ & $0 \%$ \\
Lung adenocarcinoma & $96 \%$ & $3 \%$ & $1 \%$ \\
Skin cutaneous melanoma & $94 \%$ & $3 \%$ & $3 \%$ \\
\hline
\end{tabular}

CRC: Colorectal cancer (Hobbs et al. $\left.{ }^{[31]}, 2016\right)$.

Table 3. Rates of KRAS mutations in three different types of cancer (pancreatic cancer, CRC and NSCLC)

\begin{tabular}{|c|c|c|c|c|}
\hline \multirow[t]{2}{*}{ Cancer } & \multicolumn{4}{|c|}{ Mutation rates } \\
\hline & KRAS & KRAS exon 2 & KRAS exon 3 & KRAS exon 4 \\
\hline Pancreatic & $86.3 \%$ & $79.7 \%$ & $6.6 \%$ & $0.16 \%$ \\
\hline CRC & $44.2 \%$ & $37.6 \%$ & $2.63 \%$ & $3.7 \%$ \\
\hline NSCLC & $29.6 \%$ & $27.1 \%$ & $1.89 \%$ & $0.24 \%$ \\
\hline
\end{tabular}

CRC: Colorectal cancer; NSCLC: non-small cell lung cancer (AACR Project GENIE Consortium, 2017).

As far as KRAS exons 3 and 4 is concerned, the results obtained by the AACR Project GENIE Consortium describe that these mutations are significantly rarer with respect to those occurring in exon 2; indeed, they are detected in $1.15 \%$ and $0.62 \%$ of cancer patients, respectively ${ }^{[17]}$. More in detail, the mutations in exon 3 characterize $2.63 \%$ of CRC and $1.89 \%$ of NSCLC, whereas they are more diffused in pancreatic adenocarcinoma patients $(6.6 \%)^{[17]}$ [Table 3]. A different trend is observed for exon 4: these mutations are more frequent in CRC $(3.7 \%)$ compared to pancreatic adenocarcinoma $(0.16 \%)$ or NSCLC $(0.24 \%)^{[17]}$ [Table 3].

Differences among cancer types are also observed concerning the different types of mutation: the c.182A $>\mathrm{G}$ (p.Q61R) change is very frequent in pancreatic adenocarcinoma (8.33\%), at odds with lung adenocarcinoma $(0.06 \%)$ or CRC $(0.31 \%)$. The other two most common exon 3 mutations, the c.183A>C (p.Q61H) and c.182A $>\mathrm{T}$ (p.Q61L) changes, are not relevant in pancreatic cancers (less than $0.1 \%$ ) but are detected in $1.2 \%$ and $0.4 \%$ of NSCLC and $0.98 \%$ and $0.36 \%$ of CRC, respectively ${ }^{[17]}$.

Mutations in KRAS exon 4 have been registered by the AACR Project GENIE Consortium at lower frequencies compared to alterations in exon 3, with the c.436G $>C$ (p.A146P) change detected only in CRC $(0.07 \%)$ and not in NSCLC or pancreatic cancer. On the contrary, the c.436G $>$ A (p.A146T) and c.437C $>\mathrm{T}$ 
(p.A146V) mutations are still more frequent in CRC (2.42\% and $0.6 \%$, respectively) but can be found in NSCLC as well $(0.07 \% \text { and } 0.05 \% \text {, respectively })^{[17]}$.

\section{Correlation between KRAS and sidedness}

In addition to ethnic differences, the mutational status of KRAS can be different in CRC on the basis of tumor location. In particular, these differences have been investigated comparing right-sided (RCC) vs. leftsided colon cancers (LCC). Often grouped as one disease, RCC (including cancers arisen in cecum, ascending colon, and hepatic flexure) and LCC (with cancers developed in the splenic flexure, descending colon, and sigmoid colon) actually represent clinically and molecularly distinct entities with significant differences in their prognosis and treatment outcomes.

The molecular differences between RCC and LCC have recently been extensively investigated ${ }^{[38]}$. This study describes significant differences in mutational profiles between RCC and LCC and it demonstrates that the early common somatic gene mutations are associated with the selection of different subsequent genomic events in RCC compared to LCC ${ }^{[38]}$. Focusing the attention on KRAS, Salem et al. ${ }^{[39]}$ demonstrated that KRAS mutations are detected in $61 \%-71 \%$ of RCC and at a lower frequency in LCC (30\%-36\%), data also confirmed by another independent study.

In addition, these differences were also described by other investigators who included cohorts of patients from different ethnicities. In Caucasian cohorts, KRAS mutations were detected in 52\%-65\% of RCC and $35 \%-43 \%$ of LCC while, in Asian populations, in 30\%-35\% and 20\%-25\%, respectively ${ }^{[27,40,41]}$.

Furthermore, the same differences between KRAS mutation rates in LCC and RCC have been confirmed in tumors developed in adolescents and young adults (age lower than 50 years old). In this age-selected population, next-generation sequencing analyses showed higher KRAS mutations rates in RCC than LCC (respectively, $64.1 \%$ vs. $45.5 \%, P=0.035)^{[39]}$. These results may explain different responses obtained to antiEGFR therapies. Indeed, LCC patients demonstrate a longer overall survival (OS) than individuals affected by RCC.

Another stratification based on tumor location has been performed by comparing cancers arising in the colon $v s$. the rectum. In rectal cancers, KRAS alterations are observed in nearly $42 \%$ of patients, a value comparable to that of LCC and lower if compared to $\mathrm{RCC}^{[3,3,3]}$. Similar data have been obtained by investigations on Asian populations with younger age ${ }^{[27,39]}$. All the aforementioned data have been obtained by the analysis of KRAS exon 2; data from exons 3 and 4 are still lacking.

\section{CLINICAL RELEVANCE AND PROGNOSTIC/PREDICTIVE VALUE OF RAS GENE FAMILY ALTERATIONS}

Mutations in the KRAS gene are detected in approximately 25\% of all human cancers; rates are highest in pancreatic carcinoma, reaching above $80 \%$, while, in colorectal cancer, they are found in about $40 \%$ of cases. NRAS mutations are less frequent, appearing in about $7 \%$ of cases. Their role in carcinogenesis has been proven in the preclinical setting, and, despite not being usually sufficient to initiate cancer growth and progression, they contribute to the development and progression of both adenomas and adenocarcinomas $^{[42]}$. KRAS mutation prevalence reaches $30 \%$ in lung adenocarcinomas and $5 \%$ in lung squamous cell carcinomas, and it has been shown to vary between $45 \%-54 \%$ in extrahepatic and $10 \%-15 \%$ in intrahepatic cholangiocarcinomas. They can also be detected, with variable frequency, in a wide variety of other solid and hematological cancers ${ }^{[43]}$. 
Several studies have explored a possible prognostic role of KRAS mutations; however, results are not uniform across experiences, tumor types, and stages. On the other hand, KRAS mutations seem to play a role as predictive markers of treatment response, or lack thereof, in specific clinical settings. Most notably in CRC, KRAS mutations predict a lack of response to EGFR-directed treatments.

The prognostic role of KRAS remains unclear, especially for non-metastatic stage II and III CRC, and it is controversial in both microsatellite stable (MSS) and microsatellite unstable (MSI) CRC ${ }^{[44]}$. Some studies have reported a possible association between KRAS mutations and survival, but data on this topic are quite controversial.

Among the studies suggestive of KRAS as a prognostic marker, one in particular showed a high prevalence of KRAS (and BRAF) in stage II and III MSI colon cancer and, when combined, their association with poorer survival compared to cancers wild-type (wt) in both these genes. The 5-year cancer specific survival (CSS) was 93\% (95\%CI: 84\%-100\%) for patients characterized by both KRAS and BRAF wild-type genes, whereas it was $76 \%(95 \% \mathrm{CI}: 67 \%-85 \%)$ in patients with at least one mutation in KRAS or BRAF ${ }^{[45]}$.

Eklöf et al. ${ }^{[46]}$ and Nash et al. ${ }^{[4]}$ proposed a prognostic role of KRAS mutations in MSS patients. Stage I and II patients with KRAS mutations have a high mortality, with a 5 -year survival of only $55 \%$ in KRAS mutant MSS cases vs. $68 \%$ in KRAS wt MSS cases. The correlation between KRAS mutations and survival in MSS cases has not been observed in stages III and IV, where only a strong trend towards worse survival has been described ${ }^{[47]}$.

Beside OS and CSS parameters, disease-free survival (DFS) has been investigated. Sinicrope et al. ${ }^{[48]}$ reported, in a cohort of CRC not subdivided on the basis of microsatellite status, that KRAS mutations are statistically associated with poorer DFS (HR $=1.44 ; 95 \%$ CI: 1.21-1.70; $P<0.001)$.

Recently, Marco et al. ${ }^{[49]}$ explored the relationship between KRAS mutation and immune-related changes in microenvironment in CRC; KRAS mutant tumors were enriched of M2 macrophages, Treg lymphocytes, and IL-17, deemed as pro-tumorigenic immune markers, and had fewer anti-tumorigenic CD4 T-helper lymphocytes, compared to KRAS wild-type ones.

While many studies have a propensity to suggest KRAS mutations to influence survival outcome in CRC patients, others failed to confirm such an association. Roth et al. ${ }^{[50]}$ described that KRAS molecular status does not have any prognostic value regarding OS (HR $=1.05 ; 95 \% \mathrm{CI}: 0.85-1.28 ; P=0.66)$ and relapse-free survival ( $\mathrm{HR}=4.99 ; 95 \% \mathrm{CI}$ : $0.65-3.91 ; P=0.31)$. In addition, this association was not statistically relevant even when patients were stratified by stages or microsatellite status. These findings were also confirmed by other studies ${ }^{[51-56]}$. Interestingly, when KRAS mutational status was stratified with respect to the type of mutation, patients with c.38G>A (p.G13D) may show significantly shorter survival rate than those with wild-type genotype ${ }^{[56]}$. Overall, the true role of KRAS mutational status in CRC survival is still debated, and most of the scientific literature seems against its prognostic role.

\section{RAS in metastatic colorectal cancer}

EGFR-directed antibodies, namely cetuximab and panitumumab, are routinely employed in the setting of first and further line therapy for metastatic colorectal cancer, in combination with standard chemotherapy. However, EGFR membrane expression evaluated by immunohistochemistry does not correlate with treatment benefit ${ }^{[57]}$. Rather, alterations in the signaling pathways downstream of EGFR may override the therapeutic effect of EGFR inhibition; driver mutations in the RAS gene family represent one such instance 
and can lead to a detrimental effect from EGFR-directed therapies ${ }^{[58]}$. Consequently, confirmation of $R A S$ wild-type status is mandatory before the start of such treatments. Mutations most commonly occur in exon 2 (codons 12 and 13) and are less frequent in exons 3 (codons 59 and 61) and 4 (codons 117 and 146). However, current guidelines recommend that extended RAS testing exploring exons 2, 3 and 4 (and, thus, codons $12,13,59,61,117$, and 146) should be carried out, and that only patients that show pan-RAS wildtype status may be treated with anti-EGFR agents ${ }^{[5]}$. In accordance with NCCN guidelines, testing may be carried out on histological or cytological samples from either the primitive lesion or a metastasis ${ }^{[60]}$. Available platforms range $85 \%-100 \%$ sensitivity and $98 \%-100 \%$ specificity ${ }^{[42]}$. RAS mutation is considered an early event in carcinogenesis, and consequently the mutational status is usually concordant between the primary tumor and metastatic lesions ${ }^{[6]}$. More recently, liquid biopsy has been proposed as an alternative testing strategy. With appropriate testing methodologies such as BEAMing-PCR, excellent results have been obtained, with $90.4 \%$ positive agreement, $93.5 \%$ negative agreement, and $91.8 \%$ concordance with tissuebased methods ${ }^{[62,63]}$.

As stated above, tumor sidedness (origin of the primary tumor on the right- $v$ s. left-side of the colon) also appears to play a predictive role, with right-sided tumors deriving scarce benefit from anti-EGFR treatment in the first line, despite wild-type RAS status ${ }^{[64]}$. The different biology of right- $v$ s. left-sided tumors is reflected in the 2014 consensus molecular subtypes classification, which is based on gene expression profiling and identifies four subgroups: MSI immune, canonical, metabolic, and mesenchymal. Right-sided tumors present characteristics of either the MSI immune or metabolic subtype ${ }^{[65]}$.

Colorectal tumors that display pan- $R A S$ wild-type status at diagnosis may acquire $R A S$ mutations over time and the course of treatment; indeed, selective pressure from EGFR-directed therapies may favor pre-existing or newly appearing RAS mutant clones that are not sensitive to EGFR targeting. Another possible mechanism of resistance lies in the appearance of mutations in the extracellular domain of EGFR, hindering the binding of the therapeutic agent to the receptor ${ }^{[66]}$.

Upon progression to a treatment line including an EGFR targeting agent, current practice dictates a change in the chemotherapy backbone, the biological agent, or both. Once a patient shows signs of progressive disease to anti-EGFR-directed treatment, current guidelines and most regulatory agencies do not consider the option of a rechallenge, as it is expected that acquired resistance will not be reversible. However, recent data have challenged this, highlighting the role of clonal evolution in shaping tumor responsiveness to targeted agents. Goldberg et al. ${ }^{[6]]}$ demonstrated that RAS mutant clones can emerge in originally RAS wildtype CRC over the course of anti-EGFR treatment, leading to acquired resistance ${ }^{[6,67]}$.

The CRICKET trial enrolled patients who had successfully been treated with cetuximab plus chemotherapy for a duration of at least 6 months; upon progression, they were treated with bevacizumab-containing regimens. Those who experienced benefit from this second line for a duration of at least 4 months, and then progressed, received cetuximab in combination with irinotecan as a rechallenge strategy ${ }^{[68]}$. Four out of 27 patients (14.3\%) obtained a partial response (PR), and the disease control rate was 53.5\%. Median PFS and OS were 3.4 and 9.8 months, respectively. RAS mutational status was assessed by liquid biopsy before the start of the cetuximab rechallenge; RAS mutations were found in $48 \%$ of evaluable patients, and all 4 patients who obtained PR were identified as RAS wild-type. Median reported PFS was 4 months for RAS wild-type patients vs. 1.9 months for RAS-mutant ones; median OS was 12.5 months vs. 5.2 months. These results suggest that the analysis of $R A S$ status among treatment lines might help in predicting response to anti-EGFR rechallenge ${ }^{[68]}$. 
In a similar small proof-of-concept study by Gazzaniga et al. ${ }^{[69]}$, 11 patients with RAS-mutated metastatic CRC who had been treated with bevacizumab-containing regimens were enrolled, and RAS status was tested upon progression by liquid biopsy, employing the plasma-based Idylla ${ }^{\mathrm{T}}$ system. Five out of 11 patients tested negative; 4 of them obtained confirmation of RAS wild-type status by Ion Torrent-Personal Genome Machine and were treated with anti-EGFR based therapies with longitudinal circulating tumor DNA (ctDNA) monitoring. The PFS of these patients was 12, 10, and 6 months for the three that were treated in the second line and 4 months in a patient treated in the fourth line.

Overall, this study suggests that antiangiogenetic agents may be able to revert RAS-mutated tumors to wildtype status, most likely through partially selective killing of RAS-mutant tumor cells. The authors hypothesized that $R A S$-mutated cells may be more sensitive to alterations in the vascular supply secondary to antiangiogenetic treatment. Additionally, Gazzaniga et al. ${ }^{[70]}$ assessed the $R A S$ status concordance between tissue samples and plasma in 20 patients with RAS-mutant tumors; 15 patients with concordant results were then monitored by liquid biopsy over the course of antiangiogenetic treatment with the Idylla ${ }^{\text {tw }}$ platform. Eleven out of 15 concordant patients (73\%) switched to RAS wild-type after the first line. Five of them went on to receive EGFR inhibitors in the second line setting, with a significant clinical benefit, supporting the hypothesis that anti-EGFR treatment may be of benefit to originally RAS-mutated tumors that revert to wild-type status upon treatment with antiangiogenic agents, in a similar way as in patients in the CRICKET trial upon rechallenge after reversion.

Rates of RAS mutation loss may be as low as $1.6 \%-8.8 \%$, in contrast with previous reports reaching as high as $20 \%$, as demonstrated by a study conducted by MD Anderson Cancer Center ${ }^{[71]}$. Moreover, such rates may be influenced by the sensitivity of the analytic method, particularly when liquid biopsy is employed ${ }^{[7]}$. Plasma-based methods have shown promising results in RAS mutation testing in CRC, with an overall agreement between matched tumor and ctDNA samples of over 90\%, 89\%-96\% sensitivity, and $90 \%-100 \%$ specificity in many retrospective series. However, when highly sensitive tissue-based methods are used, the concordance rate can drop to $78 \%-88 \%$, with $83 \%-91 \%$ sensitivity and $70 \%-85 \%$ specificity ${ }^{[72]}$, although some studies have suggested that the detection of underrepresented clones by such techniques does not carry clinical significance ${ }^{[73]}$. Among clinicopathological variables that are known to influence the abundance of ctDNA and, consequently, the reliability of results are cancer burden, stage, tumor biology (e.g., apoptosis rate and metastatic potential), and vascularization ${ }^{[72,74-76]}$. It cannot be excluded that the use of antiangiogenetics, beside contributing to a response that possibly decrease the overall tumor burden, may also impact the remodeling of the tumor vasculature that could potentially influence tumor DNA access to systemic circulation. Some of the limitations of liquid biopsies are likely to be overcome in the near future, in part by preanalytical and analytical standardization and in part by identifying a threshold of clinical significance and the setting in which tissue- and plasma-based methods may be interchangeable. However, current data suggest that a measure of caution should still be applied.

The fluctuations in RAS mutation levels according to applied treatment are perhaps among the most evident examples of how the interplay between multiple genetic and environmental factors can influence disease course and evolution. Indeed, each individual tumor in each individual host has a unique pathogenetic history, shaped by the interactions between genetics, exposure to environmental and behavioral risk factors, immune response, applied treatments, and the microbiome; and this complex network of interactions can result in specific molecular and pathological peculiarities within a disease continuum ${ }^{[77,78]}$.

It should be noted that the existence of this network adds a layer of complexity beyond the relatively simple identification of a clear and evident association between, e.g., a mutation in a specific gene and a resulting 
prognostic phenotype or clinical outcome. Other significant instances that exemplify this complexity are represented by the identification of genetic alterations that interact with lifestyle factors of pharmacological interventions: a study by Song et al. ${ }^{[79]}$ suggested that single nucleotide polymorphism (SNP) rs4444235 at 14 q22.2 and SNP rs2423279 at 20p12.3 appear to modify the benefit from regular exercise and regular aspirin use, respectively, in terms of risk of developing CRC. Similarly, a study by Cook et al. ${ }^{[80]}$ has shown that each KRAS allele is associated with a distinct, tissue-specific comutation network, and, moreover, these associations are tissue-specific. The study, classification, and interpretation of each disease's unique peculiarities, as well as the definition of patterns and causal relationships, is likely to benefit from the systematic application of "big data" analysis methodologies, within the paradigm defined as molecular pathological epidemiology ${ }^{[77,78]}$.

\section{RAS as a therapeutic target}

The frequent occurrence of RAS mutations in human cancers, along with the relevance of its role either as a driver mutation [as is the case for KRAS c.34G>T (p.G12C)] or in the development of treatment resistance as exemplified by colorectal cancer, makes it an attractive target for drug development in principle. Multiple approaches have been attempted in order to obtain clinically relevant inhibition of KRAS; however, most strategies until recently have been plagued by unsuccess ${ }^{[81,82]}$.

Direct RAS inhibition was first attempted by identifying molecules that could preferentially bind the RASGTP pocket. This approach was unsuccessful, possibly due to the extremely high affinity between RAS and $\mathrm{GTP}^{[83]}$. Consequently, alternative targeting strategies have been explored.

Inhibition of RAS protein expression by employing antisense oligonucleotides was successfully explored in the preclinical setting. Ross et al. ${ }^{[84]}$ tested the compound AZD4785 in mouse models of NSCLC and primates, showing marked selective depletion of KRAS protein with downstream inhibition, and in the absence of feedback activation of the MAPK pathway, which had been observed with other targeting strategies.

Similarly, Gray et al. ${ }^{\left[{ }^{[5]}\right]}$ attempted HRAS targeting by antisense oligonucleotides (ISIS 2503) in HRAS mutant cells and obtained a $90 \%$ reduction in protein expression; clinical testing followed in the setting of phase I and II studies, including one involving CRC patients and one involving pancreatic cancer patients; however, clinical activity was globally unsatisfactory and development was not pursued further ${ }^{[85-89]}$.

An alternative strategy involves the inhibition of post-translational modifications, namely prenylation, characteristic of RAS molecules that are implied in membrane localization and activity. These modifications require the addition of either a farnesyl or geranylgeranyl group, catalyzed by specific enzymes. The compound tipifarnib is a farnesyl transferase inhibitor whose activity was tested in various phase II trials on patients with different solid tumors with unsatisfactory results; phase III trials were carried out in patients with advanced CRC and as a maintenance strategy in AML, however no survival benefit was detected in either setting ${ }^{[89-92]}$.

Lonafarnib, another drug in the same class, showed insufficient activity in CRC, urothelial cancer, and NSCLC $^{[93-95]}$. It has been suggested that the failure of this line of attack might be due to the fact that KRAS and NRAS can alternatively undergo either farnesylation or geranylgeranylation, thus negating benefit from the inhibition of a single pathway. The discovery that HRAS, on the other hand, is solely dependent on farnesylation has led to the expectation that farnesyl transferase inhibitors should be active in patients with HRAS mutant cancers, and initial results from a phase II trial testing this strategy are encouraging ${ }^{[96]}$. At the 
same time, a geranylgeranyl transferase inhibitor has been developed, but clinical testing revealed a lack of efficacy as monotherapy, and dual inhibition appears to be too toxic ${ }^{[97-99]}$.

A third possible step in the prenylation process is represented by carboxymethylation, catalyzed by the enzyme isoprenylcysteine carboxylmethyltransferase (ICMT); in this regard, ICMT inhibition is being investigated as a therapeutic strategy ${ }^{[100-103]}$.

Due to these difficulties, efforts were made to target signaling downstream of RAS, through the inhibition of its effectors RAF, MEK, and ERK. Targeting of this cascade has proven a complex endeavor.

BRAF inhibition was first attempted with sorafenib, a tyrosine kinase inhibitor, and the drug has entered the therapeutic armamentarium against different malignancies, including hepatocellular carcinoma, renal cell carcinoma, gastrointestinal stromal tumors, and thyroid carcinoma ${ }^{[104-106]}$. Nevertheless, sorafenib is not considered a specific or potent BRAF inhibitor, and it has been demonstrated that its activity is likely due to multikinase inhibition $^{[107]}$.

Second generation BRAF inhibitors, such as vemurafenib, dabrafenib, and encorafenib, have shown relevant clinical activity, either alone or in combination with MEK inhibitors, particularly in cancers carrying the c. $1799 \mathrm{~T}>\mathrm{A}$ (p.V600E) mutation; most notably, such a strategy is routinely applied in melanoma, but has also shown favorable results in other settings ${ }^{[108-110]}$. Recently, results from the BEACON trial have shown that the combination of encorafenib with the anti-EGFR inhibitor cetuximab, with or without the addition of the MEK inhibitor binimetinib, is effective in BRAF c.1799T>A (p.V600E) mutant CRC with a survival advantage over irinotecan-based chemotherapy plus cetuximab (9.3 months $v s .5 .9$ months) ${ }^{[111]}$.

However, several issues remain unsolved. Mechanistically, it was expected that BRAF inhibitors could prove effective in BRAF wild-type, KRAS mutant cancers, given its downstream localization in the pathway; however, it has been observed that this strategy leads to a paradoxical increase of ERK signaling activity. This can be explained by taking into account that RAF proteins tend to dimerize, and binding of an inhibitor to one protomer induces a conformational change resulting in activation of the other, in a process defined as negative cooperativity - thus, for instance, a BRAF/CRAF heterodimer can increase ERK signaling through CRAF activation ${ }^{[112-114]}$. This plays a pivotal role in the development of secondary malignancies, which represent a known side effect of BRAF inhibitors, and it is also relevant in drug resistance that generally ensues over the course of treatment. Indeed, paradoxical ERK activation can also occur in BRAF mutant cancers. An excessive ERK signaling is usually lethal to cells, thus prolonged drug exposure can favor the emergence of BRAF-inhibition addicted cell clones with low intrinsic ERK signaling that require the paradoxical ERK activation for growth and can be responsive to a treatment holiday ${ }^{[15,116]}$.

To date, two strategies are being explored to address this issue by impeding hetero- or homodimerization: the development of pan-RAF inhibitors, which can overcome CRAF-mediated resistance by binding both protomers, and the so-called "paradox breakers", which induce a conformational change in the RAF dimer interface ${ }^{[112]}$. However, another approach challenges bypassing the paradoxical effect by direct targeting of MEK and ERK. While some MEK inhibitors (binimetinib, cobimetinib, and trametinib) are already employed in the treatment of BRAF mutated cancers, ERK inhibitors, on the other hand, have not yet transitioned into clinical practice and suffer from the hindrance of a lower therapeutic index, due to significant inhibition of the RAS/RAF/MEK/ERK pathway in healthy cells $s^{[117]}$. 
More recently, a direct approach to RAS targeting has come back to the forefront, as covalent inhibitors were developed targeting specific RAS mutations. The covalent inhibitor sotorasib, specifically targeted at the KRAS c.34G>T (p.G12C) mutation, is able to bind KRAS and lock it in an inactive state, thus suppressing downstream ERK phosphorylation. After favorable preclinical results, a phase I trial (CodeBreaK 100) was carried out in 129 patients with heavily pretreated solid tumors harboring the KRAS c. $34 \mathrm{G}>\mathrm{T}$ (p.G12C) mutation. The response rate was $32.2 \%$ in NSCLC patients, with $88.1 \%$ disease control rate and a median progression-free survival (mPFS) of 6.3 months. Specifically, in CRC patients, the response rate was $7.1 \%$ and DCR was $73.8 \%$ with a mPFS of 4 months, suggesting that KRAS c.34G>T (p.G12C) does not represent the only oncogenic driver in these patients. Indeed, Amodio et al. ${ }^{[118]}$ compared in vitro response to sotorasib in CRC- and NSCLC-derived, c.34G>T (p.G12C) mutated cell lines. They observed that downstream ERK inhibition obtained with sotorasib monotherapy is not sustained in CRC. Additionally, CRC in vitro models, unlike NSCLC models, retain sensitivity to upstream growth factor stimulation mediated by receptor tyrosine kinases, mainly EGFR, which can interfere with c.34G>T (p.G12C) blockade. Based on these data, a dual inhibition strategy was explored, revealing that not only did cetuximab sensitize c.34G>T (p.G12C) CRC cell lines to sotorasib, but, vice versa, the same combination strategy also reverted c.34G>T (p.G12C)-mediated secondary resistance to anti-EGFR antibodies ${ }^{[118]}$.

Within the CodeBreaK 100 trial, responses were also observed with sotorasib monotherapy in patients with pancreatic, endometrial, and appendiceal cancers and melanoma; and tolerance was good, with Grade 3 or 4 treatment-related toxicities occurring in $11.6 \%$ of patients ${ }^{[119]}$. Aside from the clinical relevance of these results, requiring corroboration in larger trials, these data were enthusiastically hailed as the first demonstration that RAS is, indeed, a druggable target. Nonetheless, the usefulness of sotorasib is limited to cancers harboring the c.34G>T (p.G12C) mutation. Similar to BRAF inhibitors, combination strategies are being investigated ${ }^{[120]}$. These may involve multitarget inhibition of the EGFR/RAS/RAF/MEK/ERK pathway, with the addition of an EGFR inhibitor, a MEK inhibitor, or both, or an Src homology containing protein tyrosine phosphatase 2 (SHP2) allosteric inhibitor; other approaches include associating mTOR or cyclindependent kinase targeting and immune checkpoint inhibition, with or without with the addition of cytotoxic chemotherapy.

Another recently developed covalent KRAS c.34G>T (p.G12C) inhibitor is adagrasib, whose activity is being investigated within the phase I/II KRYSTAL-1 trial. Interim results were presented in the form of abstract at the American Society of Clinical Oncology 2020 meeting $^{[122]}$. In total, 110 patients with advanced solid tumors and a KRAS c.34G>T (p.G12C) mutation, who had already exhausted standard treatment lines, received treatment with adagrasib; similar to what was reported in the CodeBreaK 100 trial, the best results were observed in the 51 patients with a NSCLC diagnosis, who reached $45 \%$ objective response rate (ORR) and $96 \%$ disease control rate (DCR), while toxicity was evaluated on all participants, with only two instances of serious adverse events (namely, hyponatremia). Data concerning 18 participants with CRC were also presented, with $17 \%$ ORR and $94 \% \mathrm{DCR}^{[122]}$. As with sotorasib, combination strategies associating adagrasib with other agents, such as checkpoint inhibitors, anti-EGFR antibodies or TKIs, or SHP-2 inhibitors, are being explored ${ }^{[123,124]}$.

\section{CONCLUSION}

Alterations involving RAS proteins and their pathway play a significant role in the biology of various cancer subtypes, as oncogenic drivers, prognostic and predictive markers, and possible targets. The high prevalence of RAS mutations across cancer types makes it an interesting therapeutic target. However, RAS is situated at the center of a complex signaling network whose redundancy and interconnection with other pathways offer significant protection from most inhibition strategies. Several different approaches are being pursued 
in the attempt to overcome these difficulties, either by targeting multiple proteins in the pathway or by combining RAS-directed treatments with different classes of agents, such as chemotherapy and immunotherapy, and more recently by direct targeting of specific KRAS mutations. The development of new therapeutic agents such as sotorasib and adagrasib possibly represents the first crack in the wall of KRAS undruggability. Although their purpose of application is limited to a specific mutation, and their precise role in the therapeutic landscape is yet to be defined, in terms of their relevance in specific cancer subtypes, usefulness as monotherapy or in one of many possible combinations, and magnitude of clinical benefit in a real-world setting. Moreover, the application of "big data" analysis techniques within the paradigm of precision medicine and molecular pathological epidemiology may improve our understanding of the role of RAS mutations in relation to other genetic and environmental factors, possibly paving the way to personalized management strategies in the setting of prevention, diagnosis, and treatment.

\section{DECLARATIONS}

\section{Authors' contributions}

Made substantial contributions to conception and design of the study and performed data analysis and interpretation: Cefalì M, Epistolio S, Palmarocchi MC, Frattini M, De Dosso S

Writing original draft: Cefali M, Epistolio S

Writing review \& editing: Cefalì M, Epistolio S, Palmarocchi MC, Frattini M, De Dosso S

Supervision: Frattini M, De Dosso S

\section{Availability of data and materials}

Not applicable.

\section{Financial support and sponsorship}

None.

\section{Conflicts of interest}

All authors declared that there are no conflicts of interest.

\section{Ethical approval and consent to participate}

Not applicable.

\section{Consent for publication}

Not applicable.

\section{Copyright}

(c) The Author(s) 2021.

\section{REFERENCES}

1. Anderson GR, Robbins KC. Rat sequences of the Kirsten and Harvey murine sarcoma virus genomes: nature, origin, and expression in rat tumor RNA. J Virol 1976;17:335-51. DOI PubMed PMC

2. Tsuchida N, Ryder T, Ohtsubo E. Nucleotide sequence of the oncogene encoding the p21 transforming protein of Kirsten murine sarcoma virus. Science 1982;217:937-9. DOI PubMed

3. Shimizu K, Goldfarb M, Perucho M, Wigler M. Isolation and preliminary characterization of the transforming gene of a human neuroblastoma cell line. Proc Natl Acad Sci U S A 1983;80:383-7. DOI PubMed PMC

4. Stratton MR, Campbell PJ, Futreal PA. The cancer genome. Nature 2009;458:719-24. DOI PubMed PMC

5. Garraway LA, Lander ES. Lessons from the cancer genome. Cell 2013;153:17-37. DOI PubMed

6. Vogelstein B, Papadopoulos N, Velculescu VE, Zhou S, Diaz LA Jr, Kinzler KW. Cancer genome landscapes. Science 2013;339:1546-58. DOI PubMed PMC

7. Fearon ER, Vogelstein B. A genetic model for colorectal tumorigenesis. Cell 1990;61:759-67. DOI PubMed

8. Ionescu DL. New approach in the pharmacologic treatment of cancer. Rev Med Chir Soc Med Nat Iasi 2004;108:509-12. PubMed

9. Vogelstein B, Kinzler KW. Cancer genes and the pathways they control. Nat Med 2004;10:789-99. DOI PubMed 
10. Kinzler KW, Vogelstein B. Cancer-susceptibility genes. Gatekeepers and caretakers. Nature 1997;386:761, 763. DOI PubMed

11. Yun J, Rago C, Cheong I, et al. Glucose deprivation contributes to the development of KRAS pathway mutations in tumor cells. Science 2009;325:1555-9. DOI PubMed PMC

12. Ying H, Kimmelman AC, Lyssiotis CA, et al. Oncogenic Kras maintains pancreatic tumors through regulation of anabolic glucose metabolism. Cell 2012;149:656-70. DOI PubMed PMC

13. Mármol I, Sánchez-de-Diego C, Pradilla Dieste A, Cerrada E, Rodriguez Yoldi MJ. Colorectal carcinoma: a general overview and future perspectives in colorectal cancer. Int J Mol Sci 2017;18:197. DOI PubMed PMC

14. Abdulkareem FB, Sanni LA, Richman SD, et a. KRAS and BRAF mutations in Nigerian colorectal cancers. West Afr J Med 2012; 31:198-203. PubMed

15. Hoffman SJ, Wu ML. Phenotypic and genotypic differences in colorectal carcinoma among Caucasians, Asians, and Hispanics lack statistical significance. Pathol Res Pract 2018;214:720-6. DOI PubMed

16. Peeters M, Kafatos G, Taylor A, et al. Prevalence of RAS mutations and individual variation patterns among patients with metastatic colorectal cancer: A pooled analysis of randomised controlled trials. Eur J Cancer 2015;51:1704-13. DOI PubMed

17. Project GENIE Consortium. AACR Project GENIE: Powering Precision Medicine through an International Consortium. Cancer Discov 2017;7:818-31. DOI PubMed PMC

18. Timar J, Kashofer K. Molecular epidemiology and diagnostics of KRAS mutations in human cancer. Cancer Metastasis Rev 2020;39:1029-38. DOI PubMed PMC

19. Palomba G, Cossu A, Paliogiannis P, et al. Prognostic role of. KRAS ;12:1415-21. DOI PubMed PMC

20. Mazurenko NN, Gagarin IM, Tsyganova IV, Mochal'nikova VV, Breder VV. The frequency and spectrum of KRAS mutations in metastatic colorectal cancer. Vopr Onkol 2013;59:751-5. PubMed

21. Shen H, Yuan Y, Hu HG, et al. Clinical significance of K-ras and BRAF mutations in Chinese colorectal cancer patients. World $J$ Gastroenterol 2011;17:809-16. DOI PubMed PMC

22. Zhu XL, Cai X, Zhang L, et al. [KRAS and BRAF gene mutations in correlation with clinicopathologic features of colorectal carcinoma in Chinese]. Zhonghua Bing Li Xue Za Zhi 2012;41:584-9. DOI PubMed

23. Zhang J, Zheng J, Yang Y, et al. Molecular spectrum of KRAS, NRAS, BRAF and PIK3CA mutations in Chinese colorectal cancer patients: analysis of 1,110 cases. Sci Rep 2015;5:18678. DOI PubMed PMC

24. Peng J, Huang D, Poston G, et al. The molecular heterogeneity of sporadic colorectal cancer with different tumor sites in Chinese patients. Oncotarget 2017;8:49076-83. DOI PubMed PMC

25. Shen Y, Wang J, Han X, et al. Effectors of epidermal growth factor receptor pathway: the genetic profiling ofKRAS, BRAF, PIK3CA, NRAS mutations in colorectal cancer characteristics and personalized medicine. PLoS One 2013;8:e81628. DOI PubMed PMC

26. Won DD, Lee JI, Lee IK, Oh ST, Jung ES, Lee SH. The prognostic significance of KRAS and BRAF mutation status in Korean colorectal cancer patients. BMC Cancer 2017;17:403. DOI PubMed PMC

27. Kumar K, Brim H, Giardiello F, et al. Distinct BRAF (V600E) and KRAS mutations in high microsatellite instability sporadic colorectal cancer in African Americans. Clin Cancer Res 2009;15:1155-61. DOI PubMed PMC

28. Kang M, Shen XJ, Kim S, et al. Somatic gene mutations in African Americans may predict worse outcomes in colorectal cancer. Cancer Biomark 2013;13:359-66. DOI PubMed PMC

29. Sylvester BE, Huo D, Khramtsov A, et al. Molecular analysis of colorectal tumors within a diverse patient cohort at a single institution. Clin Cancer Res 2012;18:350-9. DOI PubMed PMC

30. Sanchez-Ibarra HE, Jiang X, Gallegos-Gonzalez EY, et al. KRAS, NRAS, and BRAF mutation prevalence, clinicopathological association, and their application in a predictive model in Mexican patients with metastatic colorectal cancer: A retrospective cohort study. PLoS One 2020;15:e235490. DOI PubMed PMC

31. Hobbs GA, Der CJ, Rossman KL. RAS isoforms and mutations in cancer at a glance. J Cell Sci 2016;129:1287-92. DOI PubMed PMC

32. Buday L, Downward J. Many faces of Ras activation. Biochim Biophys Acta 2008;1786:178-87. DOI PubMed

33. Pylayeva-Gupta Y, Grabocka E, Bar-Sagi D. RAS oncogenes: weaving a tumorigenic web. Nat Rev Cancer 2011;11:761-74. DOI PubMed PMC

34. Genome Atlas Network. Comprehensive molecular characterization of human colon and rectal cancer. Nature 2012;487:330-7. DOI PubMed PMC

35. Kucab JE, Zou X, Morganella S, et al. A Compendium of Mutational Signatures of Environmental Agents. Cell 2019;177:821836.e16. DOI PubMed PMC

36. Fahrer J, Kaina B. Impact of DNA repair on the dose-response of colorectal cancer formation induced by dietary carcinogens. Food Chem Toxicol 2017;106:583-94. DOI PubMed

37. Hogervorst JG, de Bruijn-Geraets D, Schouten LJ, et al. Dietary acrylamide intake and the risk of colorectal cancer with specific mutations in KRAS and APC. Carcinogenesis 2014;35:1032-8. DOI PubMed

38. Imperial R, Ahmed Z, Toor OM, et al. Comparative proteogenomic analysis of right-sided colon cancer, left-sided colon cancer and rectal cancer reveals distinct mutational profiles. Mol Cancer 2018;17:177. DOI PubMed PMC

39. Salem ME, Battaglin F, Goldberg RM, et al. Molecular analyses of Left- and Right-Sided tumors in adolescents and young adults with colorectal cancer. Oncologist 2020;25:404-13. DOI PubMed PMC

40. Charlton ME, Kahl AR, Greenbaum AA, et al. KRAS testing, tumor location, and survival in patients with stage IV colorectal cancer: SEER 2010-2013. J Natl Compr Canc Netw 2017;15:1484-93. DOI PubMed PMC 
41. Gil-Raga M, Jantus-Lewintre E, Gallach S, et al. Molecular subtypes in early colorectal cancer associated with clinical features and patient prognosis. Clin Transl Oncol 2018;20:1422-9. DOI PubMed

42. Saeed O, Lopez-Beltran A, Fisher KW, et al. RAS genes in colorectal carcinoma: pathogenesis, testing guidelines and treatment implications. J Clin Pathol 2019;72:135-9. DOI PubMed

43. Uprety D, Adjei AA. KRAS: From undruggable to a druggable Cancer Target. Cancer Treat Rev 2020;89:102070. DOI PubMed

44. Gallo G, Sena G, Vescio G, et al. The prognostic value of KRAS and BRAF in stage I-III colorectal cancer. A systematic review. Ann Ital Chir 2019;90:127-37. PubMed

45. de Cuba EM, Snaebjornsson P, Heideman DA, et al. Prognostic value of BRAF and KRAS mutation status in stage II and III microsatellite instable colon cancers. Int J Cancer 2016;138:1139-45. DOI PubMed

46. Eklöf V, Wikberg ML, Edin S, et al. The prognostic role of KRAS, BRAF, PIK3CA and PTEN in colorectal cancer. Br J Cancer 2013;108:2153-63. DOI PubMed PMC

47. Nash GM, Gimbel M, Cohen AM, et al. KRAS mutation and microsatellite instability: two genetic markers of early tumor development that influence the prognosis of colorectal cancer. Ann Surg Oncol 2010;17:416-24. DOI PubMed PMC

48. Sinicrope FA, Mahoney MR, Smyrk TC, et al. Prognostic impact of deficient DNA mismatch repair in patients with stage III colon cancer from a randomized trial of FOLFOX-based adjuvant chemotherapy. J Clin Oncol 2013;31:3664-72. DOI PubMed PMC

49. Marco M, Chen C, Choi S, Pelossof R, Shia J, Garcia-aguilar J. A KRAS mutation is associated with an immunosuppressive tumor microenvironment in mismatch-repair proficient colorectal cancer. J Clin Oncol 2019;37:609.

50. Roth AD, Tejpar S, Delorenzi M, et al. Prognostic role of KRAS and BRAF in stage II and III resected colon cancer: results of the translational study on the PETACC-3, EORTC 40993, SAKK 60-00 trial. J Clin Oncol 2010;28:466-74. DOI PubMed

51. Andersen SN, Løvig T, Breivik J, et al. K-ras mutations and prognosis in large-bowel carcinomas. Scand J Gastroenterol 1997;32:629. DOI PubMed

52. Bouzourene H, Gervaz P, Cerottini J, et al. p53 and Ki-ras as prognostic factors for Dukes' stage B colorectal cancer. Eur J Cancer 2000;36:1008-15. DOI PubMed

53. González-Aguilera JJ, Oliart S, Azcoita MM, Fernández-Peralta AM. Simultaneous mutations in K-ras and TP53 are indicative of poor prognosis in sporadic colorectal cancer. Am J Clin Oncol 2004;27:39-45. DOI PubMed

54. Westra JL, Schaapveld M, Hollema H, et al. Determination of TP53 mutation is more relevant than microsatellite instability status for the prediction of disease-free survival in adjuvant-treated stage III colon cancer patients. J Clin Oncol 2005;23:5635-43. DOI PubMed

55. Hutchins G, Southward K, Handley K, et al. Value of mismatch repair, KRAS, and BRAF mutations in predicting recurrence and benefits from chemotherapy in colorectal cancer. J Clin Oncol 2011;29:1261-70. DOI PubMed

56. Dinu D, Dobre M, Panaitescu E, et al. Prognostic significance of KRAS gene mutations in colorectal cancer--preliminary study. $J$ Med Life 2014;7:581-7. PubMed PMC

57. Hecht JR, Mitchell E, Neubauer MA, et al. Lack of correlation between epidermal growth factor receptor status and response to Panitumumab monotherapy in metastatic colorectal cancer. Clin Cancer Res 2010;16:2205-13. DOI PubMed

58. Sorich MJ, Wiese MD, Rowland A, Kichenadasse G, McKinnon RA, Karapetis CS. Extended RAS mutations and anti-EGFR monoclonal antibody survival benefit in metastatic colorectal cancer: a meta-analysis of randomized, controlled trials. Ann Oncol 2015;26:13-21. DOI PubMed

59. Cutsem E, Cervantes A, Nordlinger B, Arnold D; ESMO Guidelines Working Group. Metastatic colorectal cancer: ESMO Clinical Practice Guidelines for diagnosis, treatment and follow-up. Ann Oncol 2014;25 Suppl 3:iii1-9. DOI PubMed

60. Sepulveda AR, Hamilton SR, Allegra CJ, et al. Molecular biomarkers for the evaluation of colorectal cancer: guideline from the american society for clinical pathology, college of american pathologists, association for molecular pathology, and the american society of clinical oncology. J Clin Oncol 2017;35:1453-86. DOI PubMed

61. Etienne-Grimaldi MC, Formento JL, Francoual M, et al. K-Ras mutations and treatment outcome in colorectal cancer patients receiving exclusive fluoropyrimidine therapy. Clin Cancer Res 2008;14:4830-5. DOI PubMed

62. Thierry AR, El Messaoudi S, Mollevi C, et al. Clinical utility of circulating DNA analysis for rapid detection of actionable mutations to select metastatic colorectal patients for anti-EGFR treatment. Ann Oncol 2017;28:2149-59. DOI PubMed

63. Schmiegel W, Scott RJ, Dooley S, et al. Blood-based detection of RAS mutations to guide anti-EGFR therapy in colorectal cancer patients: concordance of results from circulating tumor DNA and tissue-based RAS testing. Mol Oncol 2017;11:208-19. DOI PubMed PMC

64. Snyder M, Bottiglieri S, Almhanna K. Impact of Primary Tumor Location on First-line Bevacizumab or Cetuximab in Metastatic Colorectal Cancer. Rev Recent Clin Trials 2018;13:139-49. DOI PubMed

65. Guinney J, Dienstmann R, Wang X, et al. The consensus molecular subtypes of colorectal cancer. Nat Med 2015;21:1350-6. DOI PubMed PMC

66. Goldberg RM, Montagut C, Wainberg ZA, et al. Optimising the use of cetuximab in the continuum of care for patients with metastatic colorectal cancer. ESMO Open 2018;3:e000353. DOI PubMed PMC

67. Siravegna G, Mussolin B, Buscarino M, et al. Clonal evolution and resistance to EGFR blockade in the blood of colorectal cancer patients. Nat Med 2015;21:795-801. DOI PubMed PMC

68. Rossini D, Cremolini C, Conca E, et al. Liquid biopsy allows predicting benefit from rechallenge with cetuximab(cet)+irinotecan(iri) in RAS/BRAF wild-type mCRC patients(pts) with resistance to 1st-line cet+iri: final results and translational analyses of the CRICKET study by GONO. Ann Oncol 2018;29:v102. DOI

69. Gazzaniga P, Raimondi C, Nicolazzo C, Gradilone A, Cortesi E. ctDNA might expand therapeutic options for second line treatment 
of KRAS mutant mCRC. Ann Oncol 2017;28:v586. DOI

70. Gazzaniga P, Raimondi C, Urbano F, Cortesi E. Second line EGFR-inhibitors in RAS mutant metastatic colorectal cancer: the plasma RAS wild type "window of opportunity". Ann Oncol 2018;29:viii183-4. DOI

71. Henry J, Willis J, Parseghian CM, et al. NeoRAS: Incidence of RAS reversion from RAS mutated to RAS wild type. JCO 2020;38:180. DOI

72. Antoniotti C, Pietrantonio F, Corallo S, De Braud F, Falcone A, Cremolini C. Circulating tumor DNA analysis in colorectal cancer: from dream to reality. JCO Precision Oncology 2019;(3):1-14. DOI

73. Laurent-Puig P, Pekin D, Normand C, et al. Clinical relevance of KRAS-mutated subclones detected with picodroplet digital PCR in advanced colorectal cancer treated with anti-EGFR therapy. Clin Cancer Res 2015;21:1087-97. DOI PubMed

74. Jacobs B, Claes B, Pomella V, et al. Abstract 940: Analytical and clinical validation of the IdyllaTM ctKRAS and ctNRAS-BRAF liquid biopsy tests identifies mCRC patient groups with high and low ctDNA shedding. In: Clinical Research (Excluding Clinical Trials). Cancer Res 2018;78(13 Supplement):940. DOI

75. Diehl F, Schmidt K, Choti MA, et al. Circulating mutant DNA to assess tumor dynamics. Nat Med 2008;14:985-90. DOI PubMed PMC

76. Diaz LA Jr, Bardelli A. Liquid biopsies: genotyping circulating tumor DNA. J Clin Oncol 2014;32:579-86. DOI PubMed PMC

77. Hamada T, Keum N, Nishihara R, Ogino S. Molecular pathological epidemiology: new developing frontiers of big data science to study etiologies and pathogenesis. J Gastroenterol 2017:52,265-75. DOI PubMed PMC

78. Ogino S, Nowak JA, Hamada T, Milner DA, Nishihara R. Insights into Pathogenic Interactions Among Environment, Host, and Tumor at the Crossroads of Molecular Pathology and Epidemiology. Annu Rev Pathol 2019:14,83-103. DOI PubMed PMC

79. Song N, Lee J, Cho S, Kim J, Oh JH, Shin A. Evaluation of gene-environment interactions for colorectal cancer susceptibility loci using case-only and case-control designs. BMC Cancer 2019;19:1231. DOI PubMed PMC

80. Cook JH, Melloni GEM, Gulhan DC, Park PJ, Haigis KM. The origins and genetic interactions of KRAS mutations are allele- and tissue-specific. Nat Commun 2021;12:1808. DOI PubMed PMC

81. Degirmenci U, Wang M, Hu J. Targeting Aberrant RAS/RAF/MEK/ERK Signaling for Cancer Therapy. Cells 2020;9:198. DOI PubMed PMC

82. Khan I, Rhett JM, O'Bryan JP. Therapeutic targeting of RAS: New hope for drugging the "undruggable". Biochim Biophys Acta Mol Cell Res 2020;1867:118570. DOI PubMed PMC

83. LoRusso PM, Sebolt-Leopold JS. One step at a Time - Clinical Evidence that KRAS is indeed druggable. $N$ Engl J Med 2020;383:1277-8. DOI PubMed

84. Ross SJ, Revenko AS, Hanson LL, et al. Targeting KRAS-dependent tumors with AZD4785, a high-affinity therapeutic antisense oligonucleotide inhibitor of KRAS. Sci Transl Med 2017;9:eaal5253. DOI PubMed

85. Gray GD, Hernandez OM, Hebel D, Root M, Pow-Sang JM, Wickstrom E. Antisense DNA inhibition of tumor growth induced by cHa-ras oncogene in nude mice. Cancer Res 1993;53:577-580. PubMed

86. Cunningham CC, Holmlund JT, Geary RS, et al. A Phase I trial of h-ras antisense oligonucleotide ISIS 2503 administered as a continuous intravenous infusion in patients with advanced carcinoma. Cancer 2001;92:1265-71. DOI PubMed

87. Marshall JL, Eisenberg SG, Johnson MD, et al. A phase II trial of ISIS 3521 in patients with metastatic colorectal cancer. Clin Colorectal Cancer 2004;4:268-74. DOI PubMed

88. Alberts SR, Schroeder M, Erlichman C, et al. Gemcitabine and ISIS-2503 for patients with locally advanced or metastatic pancreatic adenocarcinoma: a North Central Cancer Treatment Group phase II trial. J Clin Oncol 2004;22:4944-50. DOI PubMed

89. Adjei AA, Mauer A, Bruzek L, et al. Phase II study of the farnesyl transferase inhibitor R115777 in patients with advanced nonsmall-cell lung cancer. J Clin Oncol 2003;21:1760-6. DOI PubMed

90. Gajewski TF, Salama AK, Niedzwiecki D, et al; Cancer and Leukemia Group B. Phase II study of the farnesyltransferase inhibitor R115777 in advanced melanoma (CALGB 500104). J Transl Med 2012;10:246. DOI PubMed PMC

91. Rao S, Cunningham D, de Gramont A, et al. Phase III double-blind placebo-controlled study of farnesyl transferase inhibitor R115777 in patients with refractory advanced colorectal cancer. J Clin Oncol 2004;22:3950-7. DOI PubMed

92. Luger S, Wang VX, Paietta E, et al. Tipifarnib as maintenance therapy in Acute Myeloid Leukemia (AML) improves survival in a subgroup of patients with high risk disease. Results of the phase III intergroup trial E2902. Blood 2015;126:1308. DOI

93. Sharma S, Kemeny N, Kelsen DP, et al. A phase II trial of farnesyl protein transferase inhibitor SCH 66336, given by twice-daily oral administration, in patients with metastatic colorectal cancer refractory to 5-fluorouracil and irinotecan. Ann Oncol 2002;13:1067-71. DOI PubMed

94. Winquist E, Moore MJ, Chi KN, et al. A multinomial Phase II study of lonafarnib (SCH 66336) in patients with refractory urothelial cancer. Urol Oncol 2005;23:143-9. DOI PubMed

95. Kim ES, Kies MS, Fossella FV, et al. Phase II study of the farnesyltransferase inhibitor lonafarnib with paclitaxel in patients with taxane-refractory/resistant nonsmall cell lung carcinoma. Cancer 2005;104:561-9. DOI PubMed

96. Ho A, Brana I, Haddad R, et al. Preliminary results from a phase 2 trial of tipifarnib in squamous cell carcinomas (SCCs) with HRAS mutations. Abstract PR08: AACR-NCI-EORTC International Conference on Molecular Targets and Cancer Therapeutics; 2019 Oct 26-30; Boston, MA, 2019.

97. Karasic TB, Chiorean EG, Sebti SM, O'Dwyer PJ. A Phase I Study of GGTI-2418 (Geranylgeranyl Transferase I Inhibitor) in Patients with Advanced Solid Tumors. Target Oncol 2019;14:613-8. DOI PubMed PMC

98. Peterson YK, Kelly P, Weinbaum CA, Casey PJ. A novel protein geranylgeranyltransferase-I inhibitor with high potency, selectivity, and cellular activity. J Biol Chem 2006;281:12445-50. DOI PubMed 
99. Lobell RB, Liu D, Buser CA, et al. Preclinical and clinical pharmacodynamic assessment of L-778,123, a dual inhibitor of farnesyl:protein transferase and geranylgeranyl:protein transferase type-I. Mol Cancer Ther 2002;1:747-58. PubMed

100. Wahlstrom AM, Cutts BA, Liu M, et al. Inactivating Icmt ameliorates K-RAS-induced myeloproliferative disease. Blood 2008;112:1357-65. DOI PubMed PMC

101. Bergman JA, Hahne K, Song J, Hrycyna CA, Gibbs RA. S-Farnesyl-Thiopropionic Acid (FTPA) Triazoles as Potent Inhibitors of Isoprenylcysteine Carboxyl Methyltransferase. ACS Med Chem Lett 2012;3:15-9. DOI PubMed PMC

102. Lau HY, Ramanujulu PM, Guo D, et al. An improved isoprenylcysteine carboxylmethyltransferase inhibitor induces cancer cell death and attenuates tumor growth in vivo. Cancer Biol Ther 2014;15:1280-91. DOI PubMed PMC

103. Wang M, Hossain MS, Tan W, et al. Inhibition of isoprenylcysteine carboxylmethyltransferase induces autophagic-dependent apoptosis and impairs tumor growth. Oncogene 2010;29:4959-70. DOI PubMed

104. Llovet JM, Ricci S, Mazzaferro V, et al; SHARP Investigators Study Group. Sorafenib in advanced hepatocellular carcinoma. $N$ Engl J Med 2008;359:378-90. DOI

105. Escudier B, Eisen T, Stadler WM, et al; TARGET Study Group. Sorafenib in advanced clear-cell renal-cell carcinoma. $N$ Engl J Med 2007;356:125-34. DOI PubMed

106. Brose MS, Nutting CM, Jarzab B, et al. Sorafenib in radioactive iodine-refractory, locally advanced or metastatic differentiated thyroid cancer: a randomised, double-blind, phase 3 trial. Lancet 2014;384:319-28. DOI PubMed PMC

107. Wilhelm SM, Carter C, Tang L, et al. BAY 43-9006 exhibits broad spectrum oral antitumor activity and targets the RAF/MEK/ERK pathway and receptor tyrosine kinases involved in tumor progression and angiogenesis. Cancer Res 2004;64:7099-109. DOI PubMed

108. Hauschild A, Grob J, Demidov LV, et al. Dabrafenib in BRAF-mutated metastatic melanoma: a multicentre, open-label, phase 3 randomised controlled trial. Lancet 2012;380:358-65. DOI PubMed

109. Subbiah V, Kreitman RJ, Wainberg ZA, et al. Dabrafenib and trametinib treatment in patients with locally advanced or Metastatic BRAF V600-mutant anaplastic thyroid cancer. J Clin Oncol 2018;36:7-13. DOI PubMed PMC

110. in multiple nonmelanoma cancers with BRAF V600 mutations; Adjuvant pertuzumab and trastuzumab in early HER2-positive breast cancer. N Engl J Med 2018;379:1585. PubMed

111. Kopetz S, Grothey A, Yaeger R, et al. Encorafenib, binimetinib, and cetuximab in BRAF V600E-mutated colorectal cancer. $N$ Engl $J$ Med 2019;381:1632-43. DOI PubMed

112. Pickles OJ, Drozd A, Tee L, Beggs AD, Middleton GW. Paradox breaker BRAF inhibitors have comparable potency and MAPK pathway reactivation to encorafenib in BRAF mutant colorectal cancer. Oncotarget 2020;11:3188-97. DOI PubMed PMC

113. Poulikakos PI, Zhang C, Bollag G, Shokat KM, Rosen N. RAF inhibitors transactivate RAF dimers and ERK signalling in cells with wild-type BRAF. Nature 2010;464:427-30. DOI PubMed PMC

114. Heidorn SJ, Milagre C, Whittaker S, et al. Kinase-dead BRAF and oncogenic RAS cooperate to drive tumor progression through CRAF. Cell 2010;140:209-21. DOI PubMed PMC

115. Wang L, Leite de Oliveira R, Huijberts S, et al. An Acquired Vulnerability of Drug-Resistant Melanoma with Therapeutic Potential. Cell 2018;173:1413-1425.e14. DOI PubMed

116. Seghers AC, Wilgenhof S, Lebbé C, Neyns B. Successful rechallenge in two patients with BRAF-V600-mutant melanoma who experienced previous progression during treatment with a selective BRAF inhibitor. Melanoma Res 2012;22:466-72. DOI PubMed

117. Cheng Y, Tian H. Current Development Status of MEK Inhibitors. Molecules 2017;22:1551. DOI PubMed PMC

118. Amodio V, Yaeger R, Arcella P, et al. EGFR Blockade Reverts Resistance to KRAS ${ }^{\mathrm{G} 12 \mathrm{C}}$ Inhibition in Colorectal Cancer. Cancer Discov 2020;10:1129-39. DOI PubMed PMC

119. Hong DS, Fakih MG, Strickler JH, et al. KRAS ${ }^{\mathrm{G} 12 \mathrm{C}}$ Inhibition with Sotorasib in Advanced Solid Tumors. N Engl J Med 2020;383:1207-17. DOI PubMed PMC

120. AMG 510 (pINN) Sotorasib Activity in Subjects With Advanced Solid Tumors With KRAS p.G12C Mutation (CodeBreak 101). Available from: https://clinicaltrials.gov/ct2/show/NCT04185883. [Last accessed on 23 Feb 2021].

121. Jänne PA, Rybkin II, Spira AI, et al. KRYSTAL-1: activity and safety of adagrasib (MRTX849) in advanced/metastatic Non-SmallCell Lung Cancer (NSCLC) harboring KRAS G12C mutation. Eur J Cancer 2020;138:S1-2. DOI

122. Johnson ML, Ou SHI, Barve M, et al. KRYSTAL-1: activity and safety of adagrasib (MRTX849) in patients with Colorectal Cancer (CRC) and other solid tumors harboring a KRAS G12C mutation. Eur J Cancer 2020;138:S2. DOI

123. Phase 2 Trial of MRTX849 Plus Pembrolizumab for NSCLC With KRAS G12C Mutation KRYSTAL-7. Available from: https://clinicaltrials.gov/ct2/show/NCT04613596. [Last accessed on 23 Feb 2021].

124. Sabari JK, Park H, Tolcher AW, et al. KRYSTAL-2: a phase I/II trial of adagrasib (MRTX849) in combination with TNO155 in patients with advanced solid tumors with KRAS G12C mutation. JCO 2021;39:TPS146. DOI 